\title{
Classic Spotlight, 1998 and 1999: Articles of Significant Interest Selected from the Journal of Virology Archives by the Editors
}

\begin{abstract}
ournal of Virology (JVI) marks its 50th year of publishing in 2017. To highlight particularly noteworthy JVI articles from over the years, 2017 issues are featuring Classic Spotlights selected from the archives by the editors. These Classic Spotlights are appearing chronologically, and in this issue, we have selected articles from 1998 and 1999.
\end{abstract}

Protection against Fatal Sindbis Virus Encephalitis by Beclin, a Novel Bcl-2Interacting Protein

The cellular antiapoptotic gene $b c l-2$ restricts viral replication and prevents virusinduced cell death. Bcl-2 blocks apoptosis induced by several different RNA viruses. In particular, many studies have focused on neurotropic RNA viruses because host mechanisms to inhibit apoptosis may be particularly important for nonrenewable neuronal cell populations in which virus-induced apoptotic death of neurons could result in irreversible central nervous system (CNS) pathology. In a previous study, Levine et al. (B. Levine, J. E. Goldman, H. H. Jiang, D. E. Griffin, and J. M. Hardwick, Proc Natl Acad Sci U S A 93:4810-4815, 1996, http://www.pnas.org/content/93/10/4810.abstract) reported that $\mathrm{BCl}-2$ protects mice against fatal encephalitis in infections with Sindbis virus, an alphavirus. To determine the mechanism by which $\mathrm{BCl}-2$ protects against Sindbis virus infection, Liang et al. (X. H. Liang et al., J Virol 72:8586-8596, 1998, http://jvi.asm.org/content/72/11/8586.abstract) performed a yeast two-hybrid screen to identify Bcl-2-interacting gene products in an adult mouse brain library. A novel 60-kDa coiled-coil protein, which the authors named Beclin, was found to interact with Bcl-2. The interaction was confirmed in mammalian cells using fluorescence resonance energy transfer microscopy. A recombinant Sindbis virus chimera that expressed full-length human Beclin was used to infect mice. Beclin overexpression in neurons in vivo inhibited Sindbis virus replication, reduced CNS apoptosis, and provided protection against fatal Sindbis virus infection. A Beclin construct lacking the putative $\mathrm{BCl}-2$-binding domain provided no protection. Thus, these findings identified a novel protein, Beclin, which through its interaction with Bcl-2 plays a role in host defense against Sindbis virus infection.

\section{Self-Inactivating Lentivirus Vector for Safe and Efficient In Vivo Gene Delivery}

Retroviral vectors have been developed as tools for human gene therapy because they stably integrate into the chromosomes of their host cells, which is important for long-term expression. Further, they have a relatively large cloning capacity. Lentivirus vectors have an additional advantage because they can transduce nondividing cells, a critical asset for some potential targets of gene therapy, such as the brain and liver. Vectors derived from human immunodeficiency virus type 1 (HIV-1) allow for efficient in vivo delivery, integration, and stable expression of transgenes into cells such as neurons and hepatocytes; however, biosafety of HIV-based vectors is an essential consideration. Zufferey et al. (R. Zufferey et al., J Virol 72:9873-9880, 1998, http://jvi .asm.org/content/72/12/9873.abstract) described a self-inactivating HIV-1 vector with a 400-nucleotide deletion in the $3^{\prime}$ long terminal repeat (LTR), which includes the TATA
Citation American Society for Microbiology 2017. Classic Spotlight, 1998 and 1999: Articles of significant interest selected from the Journal of Virology archives by the editors. J Virol 91:e00852-17. https://doi.org/ 10.1128/JVI.00852-17.

Copyright $\odot 2017$ American Society for Microbiology. All Rights Reserved. 
box. This deletion abolished the LTR promoter activity but did not affect transgene expression in vitro. Further, the self-inactivating vector transduced neurons in vivo as efficiently as a vector with full-length LTRs. This improved the biosafety of HIV-derived vectors because it reduces the likelihood that replication-competent retroviruses will originate in the vector producer and target cells. In addition, removing LTR sequences that were previously associated with transcriptional interference and suppression in vivo allows the construction of more-stringent tissue-specific vectors under the control of cellular promoters that can be regulated.

The Surface Glycoproteins of H5 Influenza Viruses Isolated from Humans, Chickens, and Wild Aquatic Birds Have Distinguishable Properties

Global outbreaks of human influenza arise from influenza A viruses with novel neuraminidase (NA) and/or hemagglutinin (HA) molecules to which humans have no immunity. The pandemic strains arise from viruses of wild aquatic birds by interspecies transmission of the whole virus or by genetic reassortment between avian and human viruses. In 1997, 18 Hong Kong residents were infected with H5N1 influenza viruses, which were closely related to $\mathrm{H} 5 \mathrm{~N} 1$ avian viruses that had recently caused influenza outbreaks in chickens in Hong Kong. Mastrovich et al. (M. Matrosovich, N. Zhou, Y. Kawaoka, and R. Webster, J Virol 73:1146-1155, 1999, http://jvi.asm.org/content/73/2/ 1146.abstract) compared the receptor-binding properties (HA) and neuraminidase activities (NA) of the human and chicken $\mathrm{H} 5 \mathrm{~N} 1$ isolates from Hong Kong with $\mathrm{H} 5 \mathrm{~N} 3$ and H5N1 viruses from wild aquatic birds. Although HAs of avian viruses bind to Sia2-3Galterminated sialylglycoconjugates and not to Sia2-6Gal-containing receptors, and human viruses have the opposite receptor binding specificity, all H5N1 viruses including the human isolate bound to Sia2-3Gal-containing receptors but not to Sia2-6Galcontaining receptors. This finding demonstrated that the receptor specificity of avian influenza viruses may not restrict initial avian-to-human transmission. The H5N1 chicken viruses differed from $\mathrm{H} 5$ viruses of wild aquatic birds by a 19-amino-acid deletion in the stalk of the NA and the presence of a carbohydrate at the globular head of the HA. These findings indicated that changes in both HA and NA may be required for the adaptation of influenza viruses from wild aquatic birds to domestic chickens and raised the possibility that chickens may be an intermediate host in zoonotic transmission.

\section{Rescue of Influenza A Virus from Recombinant DNA}

Fodor et al. (E. Fodor et al., J Virol 73:9679-9682, 1999, http://jvi.asm.org/content/ $73 / 11 / 9679$.abstract) developed a plasmid-based reverse genetics technique to facilitate the generation of recombinant influenza viruses containing specific mutations. The genome of influenza A virus consists of eight segments of single-stranded, negativesense RNA. Previously, reverse genetics for negative-strand RNA viruses relied on intracellular reconstitution of ribonucleoprotein (RNP) complexes from RNA and the viral nucleoprotein and RNA-dependent RNA polymerase, which are introduced into cells by a variety of techniques. In this study, influenza A virus was rescued by transfection of 12 plasmids into Vero cells. The eight individual negative-sense genomic viral RNAs were transcribed from plasmids containing the human RNA polymerase I promoter and hepatitis delta virus ribozyme sequences. The three influenza virus polymerase proteins and the nucleoprotein were expressed from protein expression plasmids. The system is entirely plasmid driven and does not involve the use of any helper or heterologous virus. 